\title{
Acknowledgement to Reviewers of Veterinary Sciences in 2015
}

\author{
Veterinary Sciences Editorial Office \\ MDPI AG, Klybeckstrasse 64, CH-4057 Basel, Switzerland; vetsci@mdpi.com \\ Published: 27 January 2016
}

The editors of Veterinary Sciences would like to express their sincere gratitude to the following reviewers for assessing manuscripts in 2015.

We greatly appreciate the contribution of expert reviewers, which is crucial to the journal's editorial decision-making process. Several steps have been taken in 2015 to thank and acknowledge reviewers. Good, timely reviews are rewarded with a discount off their next MDPI publication. By creating an account on the submission system, reviewers can access details of their past reviews, see the comments of other reviewers, and download a letter of acknowledgement for their records. We are grateful to our reviewers for the contribution they make.

Andre, Catherine
Arthur, Helen
Baeumer, Wolfgang
Barnhart, Kirstin
Beineke, Andreas
Bennett, Peter
Berry, Donagh P
Bertoni, Prof. Giuseppe
Bloom, Paul
Boothe, Dawn Merton
Bradford, Barry
Briner, Wayne
Bryant, Juliet
Cannon, Claire M
Cannon, Claire M.
Chaves, Raquel
Ciccarelli, Michele
Constantino-Casas,
Fernando
De Rensis, Fabio
Dobschuetz, Sophie von
Duato, Esperanza Gomez-Lucia
Duesbury, Nick
Elder, John H.
Fan, Timothy M.
Fox, Larry
Gallagher, Alexander E.
Geyer, Joachim
Goga, Beniamino Terzo Cenci
Grassi, Felice Roberto
Grasso, Felicia

Groner, Bernd

Günther, Sebastian

Halsey, Charles H. C. Hendricks, Joan C.

Hogan, Joseph

Holmes, Mark

Horvath, György

Inobe, Manabu

Jayne, David

Kania-Korwel, Izabela

Knapp, Deborah

Krimer, Paula M.

Lakritz, Jeffrey

Liao, Albert T.

Lopez Gatius, Fernando

Mackin, Andrew

Mani, Rinosh

Maniscalco, Lorella

Markey, Bryan

Materniak, Magdalena

Matsuda, Hiroshi

McCleary-Wheeler,

Angela L.

Meinecke-Tillmann,

Sabine

Milovancev, Milan

Oh, S. Paul

Ohta, Hiroshi

Page, Rodney

Passler, Thomas

Pelletier, Guillaume
Pereira, Marcos Neves

Petrovski, Kiro

Pinchuk, Lesya

Poglayen, Giovanni

Poli, Alessandro

Poroyko, Valeriy

Pourquier, Philippe

Queiroga, Felisbina Luisa

Rebhun, Robert

Rodriguez, Carlos

Rosol, Thomas J.

Sakai, Hiroki

Scherk, Margie

Sehgal, Inder

Sierra, Eva

Song, Chang-Seon

Steffen, David

Stein, Tim

$\mathrm{Su}, \mathrm{Hua}$

Suter, Steven

Tardy, Florence

Tervaert, Jan Willem Cohen

Thamm, Douglas H.

Verma, Ashutosh

Villinger, Francois

von Euler, Henrik

Walter, Ingrid

Wendt, Anna-Maria

Westman, Mark

White, Stephen

Young, Howard 
(C) 2016 by the authors; licensee MDPI, Basel, Switzerland. This article is an open access article distributed under the terms and conditions of the Creative Commons by Attribution (CC-BY) license (http://creativecommons.org/licenses/by/4.0/). 